\section{AB0090 N-ACETYL-L-CYSTEINE (NAC) CONTROLS OSTEOCLASTOGENESIS THROUGH REGULATING TH17 DIFFERENTIATION AND RANKL PRODUCTION IN RHEUMATOID ARTHRITIS}

H.-R. Kim, K.-A. Lee, S.-H. Lee. Rheumatology, Konkuk University School of Medicine, Seoul, Korea, Republic Of

Background: NAC is a thiolic antioxidant produced by the body and serves as a precursor of glutathione synthesis. In rheumatoid arthritis (RA), oxidative stress is an important mechanism causing destructive proliferative synovitis.

Objectives: This study aimed to determine the regulatory role of $\mathrm{N}$-AcetylL-cysteine (NAC), an antioxidant, in IL-17-induced osteoclast differentiation in RA.

Methods: After RA synovial fibroblasts were stimulated by IL-17, the expression and production of RANKL was determined by real-time PCR and ELISA. Human peripheral blood monocytes were cultured with M-CSF, IL-17, RANKL, and/or various concentrations of NAC, followed by counting of the cells for tartrate-resistant acid phosphatase activity to determine osteoclast formation. Osteoclastogenesis was also determined after cocultures of IL-17-stimulated RA synovial fibroblasts, Th17 cells and various concentrations of NAC with monocytes. After human peripheral CD4 ${ }^{+} \mathrm{T}$ cells were cultured with NAC under Th17 condition, IL-17, IFN-g, IL-4, Foxp3, RANKL and IL-2 expression and production was determined by flow cytometry or ELISA.

Results: When RA synovial fibroblasts were stimulated by IL-17, IL-17 stimulated the production of RANKL, and NAC reduced the IL-17-induced RANKL production in a dose-dependent manner. NAC decreased IL-17-activated phosphorylation of mTOR, JNK and IkB. When human peripheral blood CD14 ${ }^{+}$monocytes were cultured with M-CSF and IL-17 or RANKL, osteoclasts were differentiated, and $\mathrm{NAC}$ reduced the osteoclastogenesis. After human peripheral $\mathrm{CD} 4^{+} \mathrm{T}$ cells were co-cultured with IL-17-pretreated RA synovial fibroblasts or Th17 cells, NAC reduced their osteoclastogenesis. Under Th17 polarizing condition, NAC decreased Th17 cell differentiation and IL-17 and RANKL production.

Conclusions: NAC inhibits the IL-17-induced RANKL production in RA synovial fibroblasts and IL-17-induced osteoclast differentiation. NAC also reduced Th17 polarization. NAC could be a supplementary therapeutic option for inflammatory and bony destructive processes in RA.

Acknowledgements: This research was supported by a grant of the Basic Science Research Program through the National Research Foundation of Korea (NRF) funded by the Ministry of Education, Science and Technology, Republic of Korea (NRF-2014R1A2A2A01007223) and the Korea Health Technology R\&D Project through the Korea Health Industry Development Institute (KHIDI), funded by the Ministry of Health \& Welfare, Republic of Korea (HI13C1704).

Disclosure of Interest: None declared

DOI: 10.1136/annrheumdis-2017-eular.1506

\section{AB0091 SURFACE PHENOTYPE OF CIRCULATING PLATELETS IS ALTERED IN PATIENTS WITH RHEUMATOID ARTHRITIS}

H. Yasuoka, K. Yoshimoto, K. Yamaoka, T. Takeuchi. Division of Rheumatology, Department of Internal Medicine, Keio University School of Medicine, Tokyo, Japan

Background: Rheumatoid arthritis (RA) is a connective tissue disease and characterized with multiple synovitis. However, pathogenesis of RA and systemic characteristic of RA is still under the investigation (1). Platelets distribute systemically through blood circulation and classically contribute to hemostasis physiologically (2). On the other hand, platelets can contribute to disease process by producing humoral factors such as cytokines or growth factors (3). Also, platelets have surface molecules not only associated with hemostasis but with other functional properties. Thus, these molecules can trigger the activation of other cells by cell-to-cell contact (4).

Objectives: To examine the association between characteristics of phenotype of circulating platelets based on the expression of surface molecules and clinical characteristics of RA and to seek the possibility as novel biomarkers.

Methods: Eight patients with RA were involved and 9 with scleroderma and 13 healthy controls were used as controls in this study. Surface expression of CD62P (P-selectin), membrane-bound TGF (transforming growth factor)beta, CD147 (emmprin), CD142 (tissue factor), CD31 (platelet endothelial cell adhesion molecule (PECAM)-1) on CD41+CD45-CD14- platelets was examined using flow cytometry. Comparison between two groups was by non-parametric Mann-Whitney U-test. Clinical parameters at blood drawing were retrospectively obtained from clinical records, and correlation between proportion of platelet subsets and clinical parameters were examined.

Results: Proportion of CD62P+ activated platelets were higher in both RA and SSc compared to $\mathrm{HC}(\mathrm{P}<0.0005, \mathrm{P}<0.0002$, respectively). Interestingly, CD147+ platelets were significantly higher in $\mathrm{RA}(\mathrm{P}<0.0004)$, whereas not only proportion of CD147+ platelets but that of TGF-beta+ platelets were higher in SSc compared to healthy controls $(P<0.0001$, respectively). In patients with $R A$, proportion of $\mathrm{CD} 62 \mathrm{P}+$ activated platelets was correlated with inflammatory markers such as CRP and ESR and markers for disease activity such as SDAI $(P<0.05$, respectively) and these proportion were decreased after treatment $(P<0.03)$. Conclusions: In patients with RA, proportion of CD62P+ and CD147+ platelets were increased compared to healthy controls. Furthermore, phenotype of platelets of RA was altered compared to SSc. Interestingly, proportion of CD62P+ platelet is associated with the inflammatory markers and disease activity of RA. These results suggest that platelets reflect disease process of RA and could be utilized as novel biomarkers.

References:

[1] Smolen JS et al. Rheumatoid arthritis. Lancet 388: 2023-2038, 2016.

[2] Bizzozero $G$ et al. Sur un nouvel element morphologue du sang chez les mammiferes et son importance dans la thombose et dans la coagulation. Arch Ital Biol 1:1-5, 1882

[3] Morrell $\mathrm{CN}$ et al. Emerging roles for platelets as immune and inflammatory cells. Blood 123:2759-2767, 2014.

[4] Van Velzen JF et al. Multicolor flow cytometry for evaluation of platelet surface antigens and activation markers. Thromb Res 130:92-8, 2012.

Disclosure of Interest: None declared

DOI: 10.1136/annrheumdis-2017-eular.4596

\section{AB0092 EFFECT OF METHOTREXATE, LEFLUNOMIDE AND HYDROXYCHLOROQUINE ON THE INSULIN RESISTANCE AND OBESITY ASSOCIATED WITH RHEUMATOID ARTHRITIS: OBESE MOUSE MODELS OF RHEUMATOID ARTHRITIS}

I. Arias de la Rosa ${ }^{1}$, S. Rodriguez-Cuenca ${ }^{2}$, Y. Jimenez-Gomez ${ }^{1}$,

P. Ruiz-Limon ${ }^{1}$, C. Perez-Sanchez ${ }^{1}$, M.C. Abalos ${ }^{1}$, E. Collantes ${ }^{1}$,

A. Vidal-Puig ${ }^{2}$, A. Escudero-Contreras ${ }^{1}$, C. Lopez-Pedrera ${ }^{1}$, N. Barbarroja ${ }^{1}$

${ }^{1}$ Rheumatology Service, IMIBIC/Reina Sofia Hospital/University of Cordoba,

Cordoba, Spain; ${ }^{2}$ Metabolic Research Laboratories, Wellcome Trust-MRC

Institute of Metabolic Science, Addenbroke's Hospital, Universidad de

Cambridge, Cambridge, United Kingdom

Background: Numerous studies have demonstrated the closely association between rheumatoid arthritis (RA) and metabolic complications such as obesity and insulin resistance. Thus, there is an urgent need for the use of therapies targeting both the activity of the disease and such metabolic disorders. Nowadays, the conventional treatment of RA consists of disease-modifying antirheumatic drugs (DMARDs) in monotherapy or combined. Yet, its beneficial/negative effect on the metabolic complications associated with cardiovascular disease prominent in RA patients is still unravelled.

Objectives: To analyze and compare the effects of methotrexate, leflunomide and hydroxychloroquine on the obesity and insulin resistance in an obese collagen-induced arthritis (CIA) mouse model.

Methods: CIA was developed in obese and lean mice. 55 C57BI/6 mice (4-5 weeks) were used. Forty-one mice were fed with high fat diet $(60 \%)$ until reaching $30 \mathrm{~g}$ (obese) (OB). Groups of study: 5 non-diseased lean mice, $9 \mathrm{ClA}$ lean mice, 5 non-diseased OB mice, 9 OB-CIA mice, 9 OB-ClA mice treated with leflunomide (10 mg/kg daily), 9 OB-CIA mice treated with methotrexate $(3 \mathrm{mg} / \mathrm{kg}$ three times/week) and $9 \mathrm{OB}-\mathrm{ClA}$ mice treated with hydroxychloroquine $(60 \mathrm{mg} / \mathrm{kg}$ daily) for 15 days. Mice were weighted and the number of total inflamed digits was recorded daily. After treatment, before 72 hours of termination, glucose tolerance test (GTT) was performed. Buffy coat, plasma and metabolic tissues (gonadal and inguinal adipose tissue, skeletal muscle and liver) were collected.

Results: CIA obese mice developed the arthritis earlier and more severe (increased number of inflamed digits) compared with CIA lean mice. Regarding the progression of the disease, the three drugs significantly reduced the number of affected joints from the second day of treatment. However, after 15 days of treatment, the therapies more effective inhibiting the generation of inflamed digits were hydroxychloroquine and methotrexate. The development of RA in both obese and lean mice did not have effect on the body weight. Among the therapies used, only the hydroxychloroquine significantly reduced the body weight after 11 days of treatment. Glucose tolerance test revealed that the area under the curve was markedly smaller in OB-CIA mice after treatment with hydroxychloroquine compared to other treatments and OB-CIA mice untreated, suggesting an improvement of insulin sensitivity. Analysis on the metabolic tissues of these mice is currently ongoing in order to completely elucidate the effect of these therapies in the metabolic state.

Conclusions: 1) Obesity accelerates the development and aggravates the outcome of the arthritis in CIA mice. 2) Among the three DMARDs administered, hydroxychloroquine promoted a beneficial effect on the metabolism of CIA obese mice, reducing body weight and improving the insulin sensitivity. These results suggest that hydroxychloroquine could be used as a valuable therapeutic strategy in RA patients to reduce the disease activity and ameliorate the metabolic complications associated.

Acknowledgements: Funded by PI2013-0191, ISCIII-FIS (CP15/00158), RD16/0012/0015 and Roche Pharma S.A

Disclosure of Interest: None declared

DOI: 10.1136/annrheumdis-2017-eular.4684 